\title{
Traduire
}

Une autre perspective sur r tr traduction

Revue française de la traduction

224 | 2011

Des outils et des nuages

\section{Des outils et des nuages}

\section{Françoise Wirth}

\section{(2) OpenEdition}

\section{Journals}

Édition électronique

URL : http://journals.openedition.org/traduire/174

DOI : 10.4000/traduire.174

ISSN : 2272-9992

\section{Éditeur}

Société française des traducteurs

\section{Édition imprimée}

Date de publication : 1 juin 2011

Pagination : 3-6

ISSN : 0395-773X

\section{Référence électronique}

Françoise Wirth, « Des outils et des nuages », Traduire [En ligne], 224 | 2011, mis en ligne le 03 février 2014, consulté le 22 septembre 2020. URL : http://journals.openedition.org/traduire/174 ; DOI : https://doi.org/10.4000/traduire.174 


\section{é d i to}

\section{Françoise Wirth}

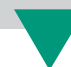

\section{Des outils et des nuages}

Outils et nuages, drôle de mélange ? Pas si incongru que cela et le lecteur le constatera rapidement dans les pages qui suivent. Avec son cahier " outils ", ce numéro de Traduire fait écho aux grandes manifestations de ces derniers mois, comme le colloque Traduction et ergonomie qui s'est déroulé à Grenoble en octobre dernier ${ }^{(1)}$ ou Tralogy ${ }^{(2)}$ plus récemment à Paris. La question de la technicité croissante de la profession est plus que jamais d'actualité. Même si, la représentation du traducteur avec crayon, gomme et gros dictionnaires papier perdure souvent dans l'imaginaire collectif(3), le quotidien du traducteur est tout différent. Et surtout, il n'arrête pas d'évoluer. Face à ces mutations plus ou moins forcées, les traducteurs ont, comme le soulignent d'entrée Caroline Champsaur et Michel Rochard dans un article issu d'une communication présentée au colloque de Grenoble, " un mélange de crainte devant la modernité (les outils vont tuer le métier) et de volonté de ne pas subir l'outil, mais de l'assujettir à leurs besoins professionnels ". Tous deux retracent pour nous, l'itinéraire parcouru par l'Unité des références et de la terminologie de l'OCDE depuis les fiches organisées dans des boîtes à chaussures jusqu'à un outil véritablement " collaboratif ". II s'agit d'une évolution remarquable à plus d'un titre car, outre l'aspect technique de l'informatisation, c'est l'approche même de la terminologie qui a changé, dépassant la démarche individualiste pour aboutir à une "appropriation de l'outil terminologique par les traducteurs, au service du collectif ". Cette nouvelle approche, permise par un " portail de la traduction " favorise non seulement le partage des références mais aussi des échanges entre traducteurs (blogs, commentaires, etc.). Clairement, les outils du traducteur ne se résument plus à ses propres outils individuels. Internet n'est

(1) Traduction et ergonomie, 15-16 octobre 2010, colloque organisé par le GREMUTS/ILCEA (Grenoble III), voir aussi le prochain numéro de la revue en ligne //cea, à paraître prochainement (http://ilcea.revues.org/).

(2) Tralogy, Métiers et technologies de la traduction : quelles convergences pour l'avenir ?, 3 et 4 mars 2011 à l'auditorium du CNRS, Paris.

(3) Un sujet que nous souhaitons aborder en 2012 avec un cahier sur l'image du traducteur. Voir en p. 15 de ce numéro la vision du traducteur " dématérialisé " de Marlène Junius. 
plus seulement l'extraordinaire source de documentation et de références que nous exploitons tous avec délice mais a pris ses dernières années une nouvelle dimension avec l'émergence du web2 et plus récemment de l'informatique " dématérialisée " et des "logiciels-services " évoqués par Anne-Marie Robert, également présente au colloque de Grenoble. La traduction entre en effet dans l'ère du cloud computing ou " informatique dans les nuages ", image bien poétique qui recouvre une réalité - non, pardon, une virtualité - qui l'est un peu moins. Appliquée à la traduction, cette technique consiste en effet à externaliser " dans les nuages ", c'est-à-dire en fait chez un fournisseur externe, une partie, voire la totalité de son système informatique. Cette nouvelle mutation aurait/aura/a bien des conséquences sur l'environnement et le mode de travail du traducteur qu'A.-M. Robert nous expose en détail. Le dernier article issu du colloque de Grenoble, nous vient de Jean-François Allain qui aborde le problème de l'organisation du travail du traducteur sous un tout autre aspect, l'informatique n'étant, naturellement, pas la seule piste ouverte à qui souhaite repenser sa manière de travailler. Fort de sa grande expérience et d'une observation aiguë du processus de traduction en action, il nous présente avec humour deux grands "types " de traducteurs et chaque lecteur pourra s'amuser à déterminer s'il ressemble davantage à un imperturbable " cheval de labour " " qui sait quand il commence et qui sait quand il aura fini " ou à un "cheval de course ", plus fougueux mais aussi plus dispersé. Sous cette catégorisation un peu moqueuse, J.-F. Allain amorce une analyse profonde du processus traductionnel et des multiples tâches qu'il implique, en faisant une distinction entre un mode de travail qui consiste à dissocier ces tâches et un autre qui consiste à les réaliser concomitamment (multitasking). L'auteur incite ainsi le lecteur à réfléchir sur son propre mode de fonctionnement et lui donne des pistes pour une meilleure organisation de son travail. L'article suivant, signé de Benjamin Phister, repousse résolument les craintes évoquées plus haut en affirmant dès son titre que l'informatique est " une aide pour le traducteur". L'auteur, bien connu pour ses formations sur la " boîte à outils du traducteur " et son rôle actif à la SFT, commence par évoquer les grandes lignes des évolutions technologiques de ces quinze dernières années avant de brosser un large panorama des principaux types d'outils informatiques disponibles, depuis la désormais incontournable traduction assistée par ordinateur (TAO) jusqu'au cloud computing, dans lequel il perçoit une sorte de clin d'œil aux gros serveurs centralisateurs d'antan, supplantés, dans l'intervalle, par l'individualisation des personal computers ou PC.

Didier Briel aborde un domaine peut-être moins connu - et certainement mal connu - des traducteurs, celui des outils dits " libres ". II commence par nous expliquer en quoi ils peuvent être considérés comme libres et souligne l'impératif de transparence qui les sépare des logiciels "propriétaires ". D. Briel passe en revue un certain nombre de ces logiciels qu'il décrit comme les "éléments d'une trousse à outils modulable en fonction des besoins et des préférences " avant d'entrer plus en détail dans le fonctionnement de l'outil de TAO Omega T, lequel a notamment comme particularité d'avoir un traducteur, et non un informaticien, comme coordinateur de projet. Un élément qui a forcément des conséquences... D. Briel aborde enfin 
l'aspect financier affirmant, un peu amèrement, que le plus grand défaut d'Omega T est sans doute sa gratuité car " comment prendre au sérieux un outil entièrement gratuit ?".

Reprenant sur un air très différent le thème " éthologique " déjà fredonné par J.-F. Allain, Céline Petit nous offre un plaisant interlude, s'attachant à faire comprendre le mode de travail d'un traducteur lambda à un conjoint imaginaire. Elle croque avec habileté les petites manies du traducteur dans les différents compartiments de sa vie professionnelle et/ou sociale. Nul doute que chacun se reconnaîtra un peu, ou beaucoup, dans cette petite musique.

Il aurait été dommage de ne pas donner, dans ce cahier, la parole à ceux qui souhaitaient faire connaître l'un ou l'autre de leurs outils préférés. Vous trouverez en page 54 et suivantes une description simple et rapide de certains de ces « incontournables ". Enfin, cette partie majoritairement technologique s'achève sur un questionnement intéressant, formulé par un confrère américain, Michael Karpa, de l'influence de l'utilisation d'internet et des outils d'aide à la traduction sur le cerveau et sur la pratique du traducteur. Voilà qui donne à réfléchir !

Toutefois, sous les nuages, que certains jugeront sombres et menaçants, qui s'amoncellent dans le ciel des traducteurs, le quotidien reste majoritairement occupé par des préoccupations linguistiques. Notre deuxième cahier aborde un domaine bien différent, celui de la transposition de l'humour dans les livres pour la jeunesse. Avec Sarah Cummins, qui fait une analyse aussi fine qu'approfondie de l'adaptation en français (mais où finit la traduction et où commence l'adaptation?) d'un titre américain pour la jeunesse, nous entrons dans une " école construite à la verticale, avec une seule salle de classe à chacun de ses 30 étages ". Ce décor qui assume par principe une part d'absurde et d'irréel est à l'image de l'humour bien particulier qui anime la version américaine. L'humour étant par définition très culturel, sa transposition dans une autre langue, et donc dans une autre culture, n'est pas évidente, et risque parfois de se faire au détriment de la trame narrative, comme le montre $S$. Cummins. Son article se dévore un peu comme une enquête policière progressant d'indice en indice, et je me garderai bien d'en dévoiler l'épilogue. Sevil Zeynaligargari et Farideh Alavi s'intéressent à un problème similaire dans un contexte tout différent puisqu'ils étudient les traductions en persan (il y en a déjà eu plusieurs !) du petit bijou de la littérature pour la jeunesse en langue française qu'est Le Petit Nicolas de René Goscinny. Ce texte est tellement ancré dans la culture française que l'on imagine aisément les multiples problèmes de traduction, et de transposition, qu'il peut poser. Les deux auteurs commencent par s'interroger sur les ressorts de l'humour dans la littérature de jeunesse avant d'entrer dans le détail de la traduction du texte proprement dit en donnant des exemples des difficultés rencontrées et des solutions retenues.

Le troisième volet de ce numéro aborde le domaine de la terminologie avec Sylvain Farge, qui analyse un aspect controversé de l'évolution de la langue française en étudiant le devenir des anglicismes. Une langue vivante est une langue qui bouge et son évolution passe par des emprunts à d'autres langues, même si cet aspect ne plaît pas à tous, et d'autant moins lorsque les " autres langues " se réduisent presque exclusivement à une seule. Quels sont donc les 
facteurs qui conduisent à utiliser des anglicismes, à les intégrer pour en faire des emprunts ou à les remplacer par des néologismes ? Le succès de ces termes est variable et $\mathrm{S}$. Farge propose des hypothèses sur les raisons de leur succès ou de leur échec en étudiant notamment le recours aux suffixes -ing et -age, respectivement. S'il n'évoque ni multitasking, ni cloud computing, c'est sans doute parce que ces emprunts sont trop récents : difficile de dire déjà ce qu'il va en advenir. Les emprunts sont loin d'être un phénomène nouveau et Michel Corbellari vient à point nommé pour nous le rappeler. $I \mid$ adopte une démarche intéressante en nous proposant une étude comparée des emprunts au latin des langues allemande et française. Ces emprunts sont nombreux, ce qui ne surprendra aucunement du français mais peut-être un peu plus de l'allemand, qui a pourtant la particularité de créer des néologismes sur le modèle des mots latins ou "faux-latinismes". Certains termes latins sont utilisés tels quels dans les deux langues, d'autres ont été traduits dans une langue et non dans l'autre et d'autres enfin sont utilisés dans les deux langues mais avec un sens différent, ce qui incitera naturellement le traducteur à une grande prudence. Nous restons encore sur le terrain de la terminologie avec Jean-Marcel Lauginie, président créateur de l'association Actions pour promouvoir le français des affaires et membre de la Commission spécialisée de terminologie et de néologie en matière économique et financière, qui nous présente " Le Mot d'Or de la Traduction francophone ", expose les étapes de sa création en 2003 et retrace son itinéraire jusqu'en 2011.

Nous avons deux comptes rendus d'ouvrages au sommaire de ce numéro. L'un, rédigé par Yvon Keromnes, porte sur un ouvrage collectif intitulé Traduire, l'art de la contrainte, qui constitue les Actes d'un colloque organisé par l'Université de Provence, et l'autre, rédigé par Nancy Mobarhan, traite d'un petit précis sur la traduction publié dans la célèbre collection Que sais-je par Michaël Oustinoff.

Nous avons aussi, comme à l'accoutumée, rendez-vous avec Maurice Voituriez et sa Rétrospective qui nous fait voyager au fil du temps. Enfin, pour ce numéro, nous avons choisi, en hommage à Pierre Agron, de poursuivre ce voyage dans le temps en publiant un extrait de la chronique que notre collègue, disparu récemment, publiait dans Traduire en 1998. Le lecteur y trouvera - déjà - un certain nombre des thèmes technologiques évoqués dans ce numéro. Dans les nuages les traducteurs? Les pieds bien sur terre, en tous cas! 